\title{
ATM LANs : New Trends and Performance Comparison
}

\author{
G. Pujolle, S.M. Jiang, Q. Sun \\ Lab. PRiSM, University of Versailles \\ 78035 Versailles Cedex, France \\ Tel: (33 1) 39254061, Fax: (33 1) 39254057
}

\begin{abstract}
Medium access techniques for very high-speed multiaccess networks (1 Gbit/s and more) are actively studied in various laboratories and standards bodies. The compatibility with the ATM technique is now required for a future homogeneity with MANs and WANs. By analysing the main ATM LANs described in the literature, this paper compares the performance of several MAC schemes. We have selected the Orwell ring with a MAC based on a slotted ring approach and a cyclic reset control mechanism. The ATMRing MAC protocol is an enhancement of the Orwell network through a new reset scheme which can achieve a less important reset overhead than Orwell. Quite different from the Orwell and ATMR networks, both DQDB and ATMi (ATM Interface) use a hybrid MAC scheme to support different classes of service, i.e., the bandwidth is divided into channels: one for the isochronous traffic and the other for the asynchronous traffic. The two sub-channels are multiplexed into the frame and may be distinguished by a special bit situated in the header of the slot. ATMi is an enhancement of RRMA (Reserved / Random Multiple Access) and DQDB-REB (DQDB with Request Balancing). ATMi supports two access modes to handle several classes of service. First mode corresponds to leased cells, based on the RRMA mechanism and the second mode provides requested cells, based on the DQDB-REB options.
\end{abstract}

\section{Keywords}

ATM LANs, Medium Access Control, Performance Comparison

\section{INTRODUCTION}

The future generation of LANs will reach the Gbit/s range. Moreover, the integration of different types of traffic on the same medium will impose constraints in terms of throughput, delay, delay dispersion, reliability and sequenced delivery. This new generation should take all of these constraints into account. In the same time, a high-speed telecommunication technology 
is emerging through B-ISDNs (Broadband Integrated Services Digital Networks). The standardization of B-ISDN follows the ATM principle (Asynchronous Transfer Mode) chosen by the ITU-T.

By choosing LANs supporting the ATM technology, the interworking process with future ATM WANs and MANs will be easy.

A large number of LANs compatible with the ATM technique have been proposed in the literature and we have selected some of them in order to compare their performance. Other high-speed LANs, like CRMA-II, MetaRing, Orbit or FFOL (performance comparison among these protocols can be found in $[1,7,13]$ ) could be chosen but we restrict ourselves to the LANs supporting a direct transfer of ATM cells.

We have selected the Orwell ring with a MAC based on the slotted ring approach and the cyclic reset control mechanism. The ATMRing MAC protocol is an enhancement of the Orwell protocol through the reset scheme which can achieve a less reset overhead than this of Orwell. Different from the Orwell and ATMR MAC protocol, both DQDB and ATMi (ATM Interface) use a hybrid MAC scheme to support different classes of service, i.e., the bandwidth is divided into two channels: one for the isochronous traffic and the other for the asynchronous traffic. Both sub-channels are multiplexed in the same frame. The sub-channels may be recognized through a special bit set in the header of the slot.

\section{ORWELL AND ATMR}

\section{Orwell}

The Orwell MAC protocol [8] is based on a slotted ring approach and a cyclic reset control mechanism. The topology of Orwell is a so-called Orwell Torus which is composed of a multiple rings configuration as shown in Figure 1.

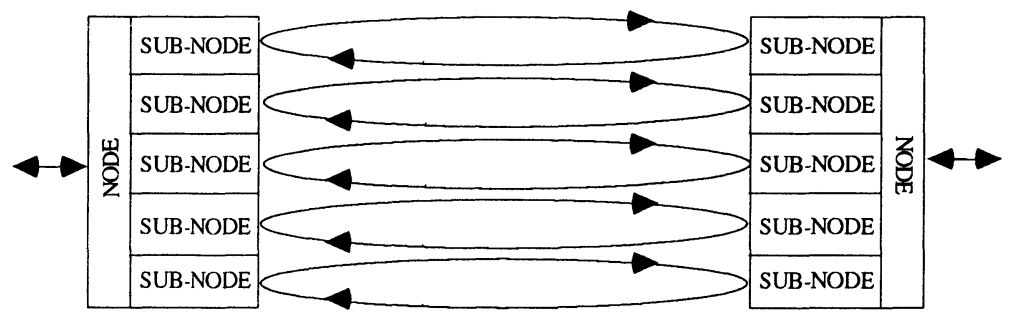

Figure 1 Topology of Orwell [2].

Each sub-node in the Torus operates the normal Orwell ring protocol for its own ring. The format of the Orwell cell is described in Figure 2. The total length of the Orwell cell may reach 58 octets when several rings are working simultaneously. The general principle can be summarized as follows:

1) A station in any ring transmits according to its window size (called D-counter) which is reset by the reset procedure. The reset procedure is issued by a temporary master station (TMS).

2) The TMS is determined in the following way: when a slot enters its destination station, this slot is released and can be re-used by this station. If this station does not use this slot for 
various reasons (zero-window size or nothing to transmit), this slot is used as the 'Trail Slot' to detect whether there is any active station on the ring by writing its own address to the 'Destination Address' field in the header of this slot. If this 'Trail Slot' returns to this station with its own address, this means that there is no active stations on the ring. Then, this station becomes the TMS.

3) The Orwell MAC protocol utilizes four levels of priority to support different classes of service. Each priority has its own queue and its own window size. When a free slot (Trail Slot) arrives, the highest priority available on the ring has the privilege to use this free slot.

4) In the sequel we assume that the transmission time of one slot corresponds to the unit of time. Two factors in the Orwell protocol will influence the performance. The first one is the interval of time between two successive reset procedures (INT), the second one is the window size $(W Z)$ assigned to the stations by the reset procedure, and there is a direct relationship between the $I N T$ and $W Z$.

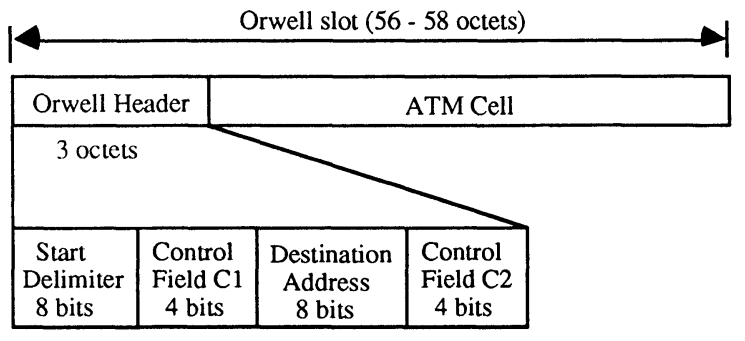

Figure 2 Format of the Orwell slot.

A practical problem is how to determine the $W Z$. When all the stations are active, the $W Z$ should guarantee each station to have an equal transmission opportunity. So, from this remark, we need a reference for the $W Z(W Z r)$, i.e., $W Z r=M / N$, where $M$ is the length of the ring (unit = slot) and $N$ is the total number of stations attached to this ring ( $N a$ denotes the number of active stations). In fact, the $W Z$ is often set larger than the $W Z r$ for a high utilization of the bandwidth since the number of active stations during a certain period of time is often less than $N$. So, the definition of the $W Z$ is not so simple.

According to the protocol, a reset procedure is issued by the TMS (temporary master station) when there is no longer active stations, i.e., when all active stations have used their allocation. So, the minimum INT (INTmin) is $(N a * W Z+M)$ because it will take some time to determine the TMS after the completion of all allocations. Since the value of $N a$ is not immediately known and varies from time to time, the INT is also variable. The expected $N a$ corresponding to a given WZ is $M / W Z$; but the real value of $N a$ fluctuates around $M / W Z$. For a real application, $N a>M / W Z$, the next reset procedure will be late; otherwise, the next reset will be early. A long $I N T$ raises the problem of guaranteeing the bandwidth and providing a bounded delay to the applications. On the other hand, the overhead of the reset procedure will cause a bandwidth wastage since there will be a gap when no transmission can occur and waiting for the reset procedure, as shown in Figure 6. Thus, a too long reset time will degrade the efficient use of the bandwidth by increasing the length of this gap. To keep a small reset rate, the $W Z$ is required to be large enough; but a larger $W Z$ will lead to a longer INT. It turns out that an efficient mechanism for determining the $W Z$ and controlling the reset procedure is required to get high performance. Hence, the protocol will become complex. Moreover, the 
expected $\mathrm{Na}$ also implies that the number of stations attached to one ring should be restricted if the length of the ring is fixed (i.e., $M$ ). It seems that the larger $M$ is, the larger $N$ could be. But if $M$ is too large, the INT will increase too much according to the INTmin mentioned above. It turns out that the network size is also required to be limited for a reasonable value of INT.

\section{ATMR - ATM Ring}

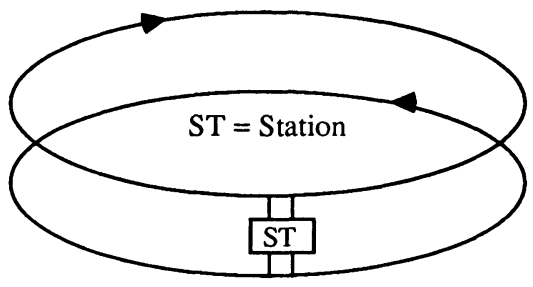

Figure 3 Topology of ATMR.

The ATMR protocol $[5,9,10]$ operates on a slotted-ring network that primarily consists of two counter-rotating rings as shown in Figure 3. The slot structure is identical to the ATM cell defined by ITU-T and is called the ATMR cell, though some subfields have different presentations to cope with the multiaccess network environment (see Figure 4).

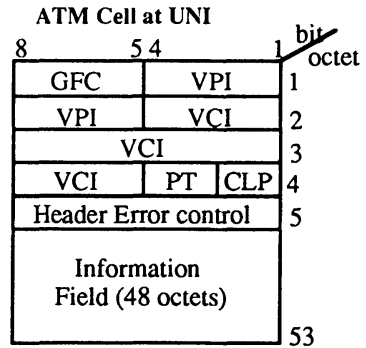

GFC: Generic flow control VCI: Virtual channel identifier CLP: Cell loss priority (1 bit)

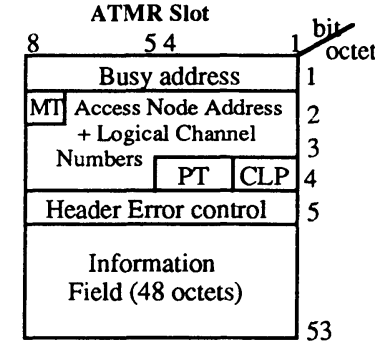

VPI: Virtual path identifier PT: Payload type (3 bits) MT: Monitor bit (1 bit)

Figure 4 ATM cell and ATMR slot.

The ATMR MAC protocol is an enhancement of the Orwell MAC from the point of view of the reset scheme which can achieve a less important reset overhead than the Orwell structure. The enhanced reset scheme is described as follows: the temporary master station (TMS) may be any station waiting for the free slot. Each awaiting station writes its address in the 'Busy address' field of passing slots. When a slot returns to a station whose address is the same as that in the 'Busy address', this station becomes the TMS, and issues the reset signal. 


\section{Analysis on Reset Overhead}

Now, we analyse the reset overhead in the ATMR and Orwell networks with a simple model of two equidistant stations (ST1 and ST2) on one ring (Figure 5). Suppose $T 1$ and $T 2$ be the propagation delay between ST1 and ST2 $(T 1=T 2)$. The unit of time for both $T$ and $W Z$ is a time slot. Suppose also ST1 is active and initialize a reset, ST2 is non active, the destination of the slots from ST1 is ST2, and WZ = 1 slot. In this case we can find the difference between the reset overhead in Orwell and ATMR as shown in the following analysis:

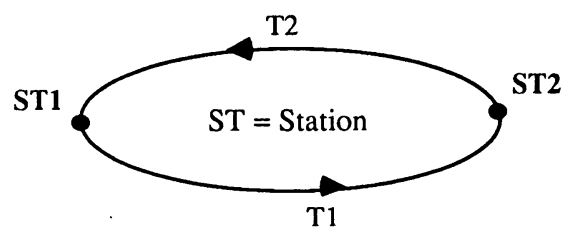

Figure 5 Configuration model of example.

We can find from the examples shown in Figure 6 and Figure 7, that in the same condition the value of $I N T$ for ST1 in Orwell (i.e., $2 *[T 1+T 2]$ ) is twice of that in ATMR (i.e., $[T 1+T 2]$ ), and the period during which no transmission occurs in Orwell is also longer than in ATMR. So, ATMR should achieve better performance than Orwell on the access delay and the throughput [5].

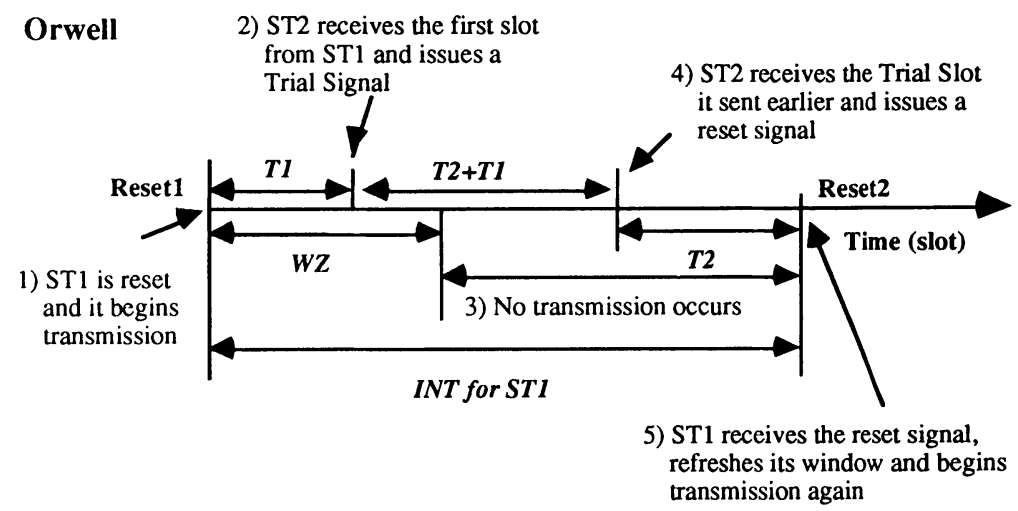

Figure 6 An example of the reset procedure in the Orwell network.

But, the reset procedure in ATMR is more complex than in Orwell when the priority is introduced since the reset procedure for different priorities is not the same one in ATMR while in Orwell one procedure can set the window for all priorities. This can be further explained as follows: in Orwell, the window for each priority is reset by the same reset procedure; but, in ATMR, there are several procedures of resetting the window size depending on the priority. Moreover, these procedures associated to the priorities may be issued at different instants. 
Another advantage of Orwell comes from the more stations attached to the network that it can support compared with ATMR due to the multiple rings configuration as shown in Figure 1. However, the length of the slot in ATMR is equal to the length of the ATM cell, without the 3 $\sim 5$ octets overhead appearing in Orwell.

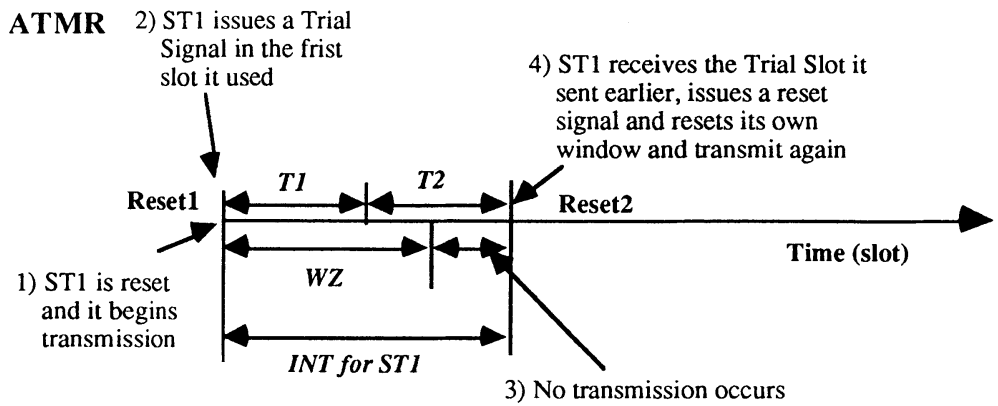

Figure 7 An example of a reset procedure in the ATMRing.

\section{DQDB AND ATMI}

Quite different from Orwell and ATMR, both DQDB and ATMi (ATM Interface) use a hybrid MAC scheme to support different classes of service, i.e., the bandwidth is divided into two sub-channels: one for the isochronous traffic and the other for the asynchronous traffic. Both sub-channels are multiplexed into the frame and may be distinguished by a special bit situated in the header of the slot. We introduce these networks in detail in the following sub-sections.

\section{DQDB - Distributed Queue Dual Bus}

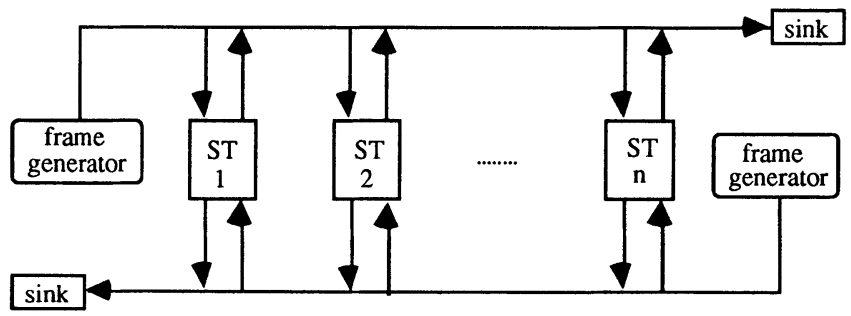

Figure 8 Topology of DQDB.

DQDB [4] is based on two unidirectional buses which operate in the opposite direction as shown in Figure 8. There are two frame generators situated at the beginning of each bus for generating the DQDB frame. DQDB uses two access modes, i.e. Queued Arbitrated (QA) to transport the connectionless data and Pre-Arbitrated (PA) for isochronous services. The QA access mode permits the station to enter the slotted (Figure 9) physical medium according to the arrival order and the priorities which are stored in a distributed queue available in each station. The PA access mode is different from the QA mode by using PA segments which are 
characterized by a specific VCI value. The assignment of the PA segments is based on a connection acceptance procedure. But the two segments (PA and QA) are multiplexed and distinguished by the slot-type bit in the ACF (Access Control Filed).

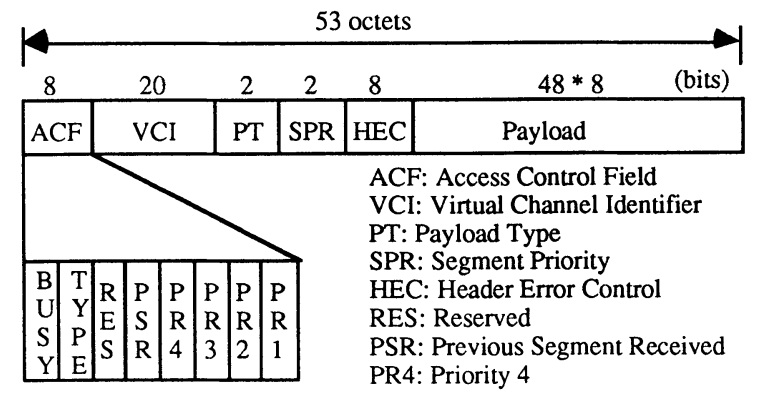

Figure 9 Format of the DQDB slot.

Since a special unit 'frame generator' is introduced, the relative position to the frame generator will differ with the location of the stations on the network. This introduces the famous fairness problem on DQDB $[6,11]$. We introduce this situation according to the two following typical cases.

DQDB can be classified into the DQDB with standby (DQDB-WS) and DQDB without standby (DQDB-NS) according to the request scheme. In both them, a station can only have an outstanding request each time. In DQDB-WS, a station can use an available slot immediately without issuing a request in the case of no other requests are in its counter. This implies that the upstream stations, which are close to the slot generator, may control the bandwidth. On the contrary, in DQDB-NS, using a slot requires issuing a request. In this case, downstream stations which are farther from the slot generator on the transmission bus will dominate the bandwidth $[11,16]$. Different papers have shown that DQDB-WS behaves better than DQDBNS such as the immediate access even in heavy traffic load; but in DQDB-NS, there is no immediate access even in light traffic load. Moreover, in DQDB-WS, any station with heavy traffic load can dominate the bandwidth even if other stations have light traffic loads. But the priority mechanism in DQDB-NS can work more efficiently than in DQDB-WS because the former respects the priority represented by the request [16].

Many proposals in the literature have been directed against the unfairness of DQDB-WS. Among them, the bandwidth balancing mechanism (BWB [3]) proposed by AT\&T Bell Laboratory has been proved efficient to alleviate the unfairness of DQDB-WS [6]. Its principle is to oblige the stations to give up a fraction of the bandwidth which they would have otherwise captured to their downstream stations. So, along with the station's working time, stations in different positions on network, or heavy users and light users, will trend to fairly share the bandwidth. This mechanism may cause a bandwidth wastage and needs a transient time to achieve the fairness. Moreover, it cannot efficiently support multi-priorities nor the transmission in contiguous slots due to the fact that it is based on DQDB-WS.

However, in some cases, the unfairness between heavy users and light users cannot be alleviated completely. For example, if high-throughput and low-throughput stations are attached to the same network, it is not reasonable to let the high-throughput station to transmit at the same speed as the low-throughput station. So, it is normal that heavy users capture more 
bandwidth than light users. So, the BWB mechanism may degrade the performance of highthroughput entities attached to the network.

We proposed another method called Request Balancing (REB [16]) to alleviate the unfairness in DQDB-NS. Its principle is to add one (or two) extra request field in each frame to provide an enough request capacity to all stations. This mechanism permits each station to have more than one outstanding request at a time, i.e., to send a bulk of requests if necessary. So, the unfairness of the original DQDB caused by the relative position of the stations on the network has been significantly alleviated by the REB mechanism, meanwhile, this allows highthroughput stations to obtain the correspondent bandwidth without the absolute dominance on the bandwidth. In addition, this modified DQDB (called DQDB-REB) can support multipriorities and the transmission in contiguous slots. The transmission in contiguous slots will provide a global response time smaller than the transmission in separated slots.

\section{ATMi - ATM Interface}

ATMi $[17,18]$ is an enhancement from RRMA (Reserved / Random Multiple Access [14, 15]) and DQDB-REB [16]. The RRMA MAC protocol uses three methods to control the access to medium corresponding to different traffics, that is, the successive reservation for delay-sensitive traffics, the once reservation for non-delay sensitive traffics and the random access mode for urgent traffics. The ATMi MAC protocol uses two access modes to support all classes of services. One is leasing cell mode based on the successive reservation mode of RRMA, and the other is requesting cell mode based on the DQDB-REB scheme (the DQDBREB is also based on the once reservation mode of RRMA). The topology of ATMi is a counter-rotating dual-ring on which the station and the frame generator are connected (see Figure 10).

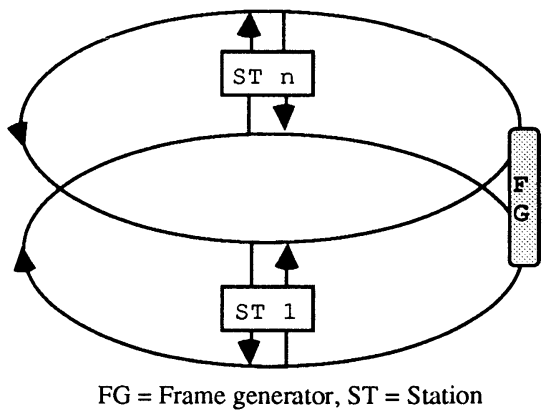

Figure 10 Topology of ATMi.

The frame generator takes charge of the generation of a frame every $125 \mu \mathrm{s}$. A frame consisting of ATMi cells is encapsulated by a header and a trailer suited at its both ends. The ATMi cell is compatible with the ATM cell at the AAL level as shown in Figure 11. The ATMi cells in each frame are divided into two, one for delay-sensitive traffics (CDS) and the other for non-delay-sensitive as well as bursty traffics (CNS). This division can be modified according to the status of network traffic at anytime. We use also the b7 bit of GFC to specify the type of the cell, and the other bits to indicate the cell state and user's requests as shown in Figure 11. 


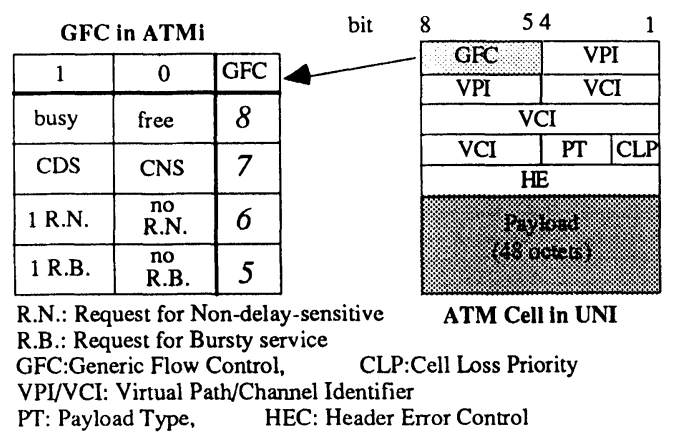

Figure 11 ACF of ATMi and GFC of the ATM cell.

The DQDB-REB based access scheme is used to control stations to share the bandwidth for non-delay-sensitive and bursty traffics. Moreover, an isochronous access mode is provided by a quick reservation scheme with which the requests of a station can be confirmed at the request time. The immediate access in this mode is also available. This scheme is realized by broadcasting the available bandwidth for delay-sensitive traffics (ABDS) to all stations through the header of the frame. When a station wants to reserve leased cells, the station first makes a comparison between the ABDS and the number of the leased cells it will request. If the ABDS is enough, the station initializes the global request which is immediately confirmed. Otherwise, the station does not initialize any request and will try again in the next arriving frame. Such a reservation can provide periodically a fixed quota of bandwidths (i.e. a number of cells in each frame) to the stations which have made this reservation for delay-sensitive traffics until the end of the transmission. So, the station must cancel its reservation when it complete the transmission. On the other hand, each cell is numbered (called $\mathrm{N}^{\circ}$ _cell) according to its order in the frame by countering the cells passing from the header to trailer of frame. The $\mathrm{N}^{\circ}$ _cell can tell each station the location of cells it used in a frame so that the station can know the corresponding cells leased to it in the next arriving frames. Thus, the delay between transmissions in two contiguous frames is fixed at the duration of a frame (e.g. 125 $\mu$ s). This is very important for guaranteeing the QoS of isochronous services. Moreover, the reservation scheme in ATMi is independent of the connection procedure unlike the PA scheme in DQDB so that this scheme is simple and quick.

Octet:
ATM cell:
Connection-oriented:
Connectionless:

\begin{tabular}{|r|c|c|c|c|c|}
\hline $\mathbf{1}$ & \multicolumn{2}{|c|}{$\mathbf{2}$} & $\mathbf{3}$ & $\mathbf{4}$ & $\mathbf{5}$ \\
\hline$G F C$ & $V P I$ & & $V C I$ & $P T \ldots$ & $H E C$ \\
\hline $10 \mathrm{xx}$ & VPI & & VCI & PT... & HEC \\
\hline $11 \mathrm{xx}$ & D_AUID & S_AUID & PT... & HEC \\
\hline
\end{tabular}

AUID: Access Unit Identifier, D: Destination, S: Source, X: 0 or 1

Figure 12 Format for the ATM cell and the ATMi cell.

To support connection-oriented and connectionless communications, we use two address formats for the ATMi cells as shown in Figure 12. For the connection-oriented mode (i.e. GFC $=10 \mathrm{xx}$ ), the field VPI/VCI has the same format as that for the ATM cell to facilitate the interconnection with B-ISDN, since the ATM based B-ISDN is connection-oriented. For the 
connectionless mode (i.e. $\mathrm{ACF}=11 \mathrm{xx}$ ), the field $\mathrm{VPI} / \mathrm{VCI}$ is divided into two parts (i.e. AUID: Access Unit Identifier, each has a length of 12 bits) in order to support connectionless transmissions in the local area. The first is the Destination AUID (D_AUID) and the second is the Source AUID (S_AUID). The D_AUID is used to route cells to their destinations in an ATMi network, and the S_AUID is used for receivers to differentiate the source of arriving cells.

\section{CONCLUSION}

The following table is presenting a comparison among protocols described in previous section.

Table 1 Comparison among above MAC protocols

\begin{tabular}{llllll}
\hline \multicolumn{7}{c}{ Non-Hybrid scheme } & \multicolumn{2}{l}{ Hybrid scheme } \\
Compared parameters & Orwell & ATMRing & DQDB & DQDB-BWB & ATMi \\
\hline Complexity of protocol & +++ & +++++ & ++ & +++ & ++ \\
Compatibility with B-ISDN & +++ & ++++ & ++++ & ++++ & +++++ \\
Network size supported & +++ & +++ & +++++ & +++++ & +++++ \\
Guarantee of bound delay & +++ & ++++ & +++++ & +++++ & +++++ \\
Bandwidth utility & ++++ & +++++ & +++ & ++ & +++ \\
Fairness of protocol & +++++ & +++++ & +++ & ++++ & ++++ \\
Efficiency of priority & +++++ & +++++ & +++ & ++ & ++++ \\
\hline
\end{tabular}

the number of ' + ' only indicates the relative order not the absolute state

From Table 1, we can find none of them is complete. From the view of point on the structure, the complexity of the Orwell and ATMR protocols results from the control of the reset procedure and the slot reutilization. Slot reutilization requires stations to hold passing slot for checking the address. So, an extra delay arises and the structure of the station becomes complex. The complexity of DQDB-BWB comes from controlling the balancing parameter for achieving the best tradeoff between the fairness and bandwidth utility.

In terms of compatibility with B-ISDN, the Orwell slot can be regarded as the 'wagon' carrying the ATM cell. The slot of ATMR and DQDB is the wagon for the 48-octet Information Payload. The ATMi slot is the ATM cell in the connection-oriented mode. The difference from the connectionless mode is the division of the VPI/VCI field into two equal length parts for supporting direct connectionless services as mentioned above.

In theory, the network size in the above hybrid schemes is unlimited; but in the non-hybrid scheme, the network size will be restricted for guaranteeing the access delay required by services asking for an isochronous flow. From this point of view, Orwell and ATMR are more suitable in a small area like LANs. On the contrary, DQDB and ATMi are well suited for large areas like MANs.

To support different classes of services like video and data, the MAC protocol must be able to guarantee the QoS on the delay and the loss required by the service. So, the bounded delay for isochronous services is one of the key aspects to measure the performance of the multimedia MAC protocol. We can find that the bounded delay for isochronous services can be guaranteed in the above hybrid schemes by the bandwidth reservation; but in non-hybrid scheme, the bounded delay can be provided through a priority mechanism. However, this priority will fluctuate with the traffic status on the network. In other words, this method cannot provide a fixed amount of bandwidth to the high-priority user since the advantage of the highpriority scheme exists only in the comparison with the low priority. This advantage will become weak if there are many high-priority users. In this scheme, a mechanism for 
controlling the number of active high-priority users is required. Of course, the reservation of bandwidth will weaken the flexibility in using the bandwidth; hence, a bandwidth wastage will occur.

The high utilization of the bandwidth in ATMR and Orwell networks is due to the circular reutilization of slots. For DQDB and ATMi, this mechanism may be an optional part. So, there are also modified versions of DQDB for having the slot reutilization to improve the bandwidth utilization through a special erasing node such as $D^{3} Q[12]$. In ATMi, the cell reutilization may also be implemented without changing the protocol. In terms of the fairness, there is a serious unfairness in the original DQDB due to the impact of the topology. So, in ATMR and Orwell there is no such problem due to their uniform topology. In the hybrid schemes, the support of various services (delay-sensitive and non-delay-sensitive) is based on the division of the bandwidth. On the contrary, in ATMR and Orwell the support of various services is based on the priority mechanism. So, the priority function in the hybrid scheme is not so strong as in the non-hybrid. And among above hybrid schemes, priority of the DQDB-BWB is shown to be the less efficient because the network cannot respect adequately the priority represented by the request.

\section{REFERENCE}

[1] A. Greb, C. Bach, "Performance Comparison of Media Access Protocols in the Gbit/s range", HighPerformance Network'94 (1994), pp 47-61.

[2] M. De Prycker, "Asynchronous Transfer Mode, Solution for Broadband ISDN", second edition (1992), p30-35

[3] E. L. Hahne, A. K. Choudhury, N. F. Maxemchuk, "Improving the Fairness of Distributed-Queue-DualBus Network", INFOCOM'90, (1990), pp 175-184.

[4] G. C. Kessler, "Standards IEEE 802.6 MAN, an Overview of this Metropolitan Area Network Standard", LAN Magazine, (April 1990).

[5] H. Ohnishi, N. Morita, S. Suzuki, "ATM Ring Protocol and Performance", ICC'89, (1989), pp 394-398.

[6] H. R. van As, "Performance Evaluation of Bandwidth Balancing in the DQDB Mac Protocol", LAN'90, (1990), pp 231-239.

[7] T. Meuser, "Performance Comparison of Media Access Protocols for Gbit/s Networks in the Local Area", 6th IEEE Workshop on LAN \& MAN, 14 - 16 October 1993 in San Diago (1993).

[8] J.L. Adams, "Orwell", Computer Networks and ISDN Systems, Vol. 26 (1994), pp 771-784.

[9] K. Imai, T. Honda, "ATMR: Ring Architecture for Broadband Networks", GLOBECOM 90, (1990), pp 1734-1738.

[10] K. Imai, T. Ito, H. Kasahara, N. Morita, "ATMR: Asynchronous Transfer Mode Ring Protocol", Computer Networks and ISDN Systems, Vol. 26, (1994), pp 785-798.

[11] K. Sauer, W. Schödl, "Performance Aspects of The DQDB Protocol", ITC Specialist Seminar, Adelaide, (1989), Paper No. 16.3.

[12] P. Davids, B. Kremer, T. Meuser, "Performance Enhancement of DQDB Networks", 20th Annual European Conference on Fibre Optic Communications and Networks, Paris, France (1992).

[13] S. Breuer, T. Meuser, "Enchanced Throughput in Slotted Rings Employing Spatial Slot Reuse", INFOCOM'94 (1994).

[14] S.M. Jiang, A. Tubtiang, H.I. Kwon, G. Pujolle, "RRMA: Reserved/Random Multiple-Access, A Candidate for ATM LAN", IFIP Workshop TC6, 1st UK Workshop on Performance Modelling and Evaluation of ATM Networks, (1993), pp 18/1-10.

[15] S.M. Jiang, G. Pujolle, "Analysis of RRMA for Topologies of Dual-bus, Folded-bus and Dual-ring", ICCT'94, Shanghai, China, (1994), pp 17.08 01- 17.08.06.

[16] S.M. Jiang, G. Pujolle, "Analysis on the Unfairness Behaviour of DQDB", PRiSM Research Report, (1994).

[17] S.M. Jiang, G. Pujolle, "Architecture and Performance of an ATM based MAC protocol for multimedia", PRiSM Research Report, 1994.

[18] S.M. Jiang, G. Pujolle, "Enhanced RRMA: Reserved Access Ring", IFIP TC6, Working Group 6.4, Second Workshop on Performance Modelling and Evaluation of ATM Networks, University of Broadford, UK (1994). 


\section{BIOGRAPHY}

Mr. Guy Pujolle received the Ph.D. and "Thèse d'Etat" degrees in Computer Science from the University of Paris IX and Paris XI on 1975 and 1978 respectively. He is currently a professor at the University of Versailles and a member of the PRiSM Laboratory. Before he was professor at the University of Paris VI and head of the MASI Laboratory depending on the CNRS (Centre National de la Recherche Scientifique) 1981-1993. Before that he was professor at ENST (Ecole Nationale Supérieure des Télécommunications), 1979-1981, and member of the scientific staff of INRIA (Institut National de la Recherche en Informatique et Automatique), 1974-1979. He is also chairman of the IFIP WG 6.4 on "Local and Metropolitan Communication Systems" and member of the IFIP WG 7.3 on "Performance Evaluation". His research interests include the analysis and modelling of data communication systems, protocols, high speed LANs and MANs and B-ISDN. He is the author of several books and of many papers on diverse aspects of performance analysis and data communication networks.

Mr. Shengming Jiang, born in November 1964 in Wuxi of China, obtained his Bachelor diploma from the computer department of Shanghai Maritime University in July 1988, then worked as a computer engineer in Nanjing Oil Transportation Company in China until the end of 1990. During his studies in France from 1991 to 1995, he obtained respectively the D.E.A. diploma of University Paris VI in September 1992 and the Ph.D. of University of Versailles in January 1995 on computer science. Now, he is a visiting scholar in the Hongkong Telecom Institute of Information Technology.

Miss Qi Sun, born in June 1962 in Beijing of China, obtained her Bachelor diploma on computer science from Beijing University of Posts \& Telecommunications in China in July 1984, then worked as a computer engineer in Beijing Science \& Technology of Development \& Exchange Centre of China till 1990. She obtained her D.E.A. diploma on computer science of University of Versailles in September 1994, and now she is working for her Ph.D. on the subject of the ATM network in the Laboratory PRiSM of University of Versailles. 